\title{
Alternativas de procesamiento de uchuva (Physalis peruviana L) para el aprovechamiento de frutos no aptos para la comercialización en fresco Gooseberry (Physalis peruviana L) processing alternatives for the profit of the fruits unsuitable for the marketing fresh
}

\author{
Processamento alternativo de groselha capa (Physalis \\ peruviana $L$ ) para o uso de frutas impróprias para \\ comercialização em fresco
}

Adriana María Castro Sánchez', Gloria Acened Puentes Montañez² \& Yolanda Botía Rodríguez ${ }^{3}$

IIngeniera de Alimentos, MBA. ${ }^{2}$ Administradora Agrícola, Especialista en Proyectos de Desarrollo, Magister en Ciencias Agrarias. ${ }^{3}$ Administrador Agrícola.

Especialista en Mercadeo Agrícola. Magister en Desarrollo Empresarial Agropecuario.

1,2,3 Escuela de Administración de Empresas Agropecuarias. Universidad Pedagógica y Tecnológica de Colombia, Facultad Seccional Duitama. Carrera 18 Calle 22. Duitama, Boyacá, Colombia.

1adriana.castro01@uptc.edu.co, ${ }^{2}$ glorispuentes@hotmail.com, ${ }^{3}$ yolandabotia3@yahoo.es

\section{Resumen}

El aprovechamiento de frutos no aptos para la comercialización en fresco, representa un área con gran potencial económico. En las regiones donde se produce uchuva en Colombia es necesario considerar y desarrollar alternativas de procesamiento de este fruto, dado que las pérdidas en cosecha y poscosecha de uchuva en el país, pueden alcanzar hasta el $45 \%$ del total de la producción. En este trabajo, se elaboró pulpa, mermelada y néctar de uchuva ecotipo Colombia con frutos rajados y blandos, provenientes de los municipios de Ventaquemada y Ciénega del departamento de Boyacá. Los resultados de este trabajo, tienen aplicaciones para las regiones productoras de uchuva en Colombia que adolecen de pérdidas de fruta por rajado y ablandamiento. Se realizaron tres formulaciones de cada subproducto, variando el contenido de pulpa en el producto final y se realizó la caracterización fisicoquímica, microbiológica y sensorial. Finalmente, se calculó el costo de cada subproducto más aceptado por el panel. Los resultados mostraron que a nivel microbiológico y fisicoquímico, los subproductos cumplen la normatividad. A nivel sensorial, la pulpa más aceptada fue la pasterizada con azúcar, el néctar mas aceptado fue la formulación con $20 \%$ de pulpa y la mermelada más aceptada fue la formulación con $50 \%$ de pulpa. El costo para procesar $1 \mathrm{~kg}$ de fruta fue de 3,11USD para pulpa, 3,36USD para néctar y 3,66USD para mermelada.

Palabras clave: uchuva, rajado, subproducto, inocuidad.

\section{Abstract:}

The use of unsuitable fruits for marketing in fresh, represents an area with great economic potential. In the regions of Colombia where is produced cape gooseberry it is necessary to consider and develop processing alternatives of this fruit, because the losses in harvest and postharvest of gooseberry in the country, can reach up to $45 \%$ 
of the total production. In this research, it was developed pulp, jam and nectar of cape gooseberry ecotype Colombia with cracked and soft fruits, from Ventaquemada and Ciénega, municipalities of the department of Boyaca. The results of this research have applications for the producing regions of cape gooseberry in Colombia that suffer from loss of fruit by cracking and softening. There were three formulations of each by-product, by varying the content of pulp in the final product and the physiochemical characterization, microbiological and sensorial. Finally, it was calculated the cost of each most accepted by-product by the panel. The results showed that at a microbiological I and physic-chemical level, the sub-products meet the regulations. At sensory level, the most widely accepted pulp pasteurized with sugar, the most accepted nectar was the formulation with $20 \%$ of pulp and the most widely accepted jam was the formulation with $50 \%$ of pulp. The cost to process $1 \mathrm{~kg}$ of fruit was 3,11 USD for pulp, 3,36 USD for nectar and 3,66 USD for jam.

Key-words: gooseberry, cracked, by-product, safety

\section{Resumo}

Aproveitando de frutos impróprios para a comercialização em fresco, representa uma área com grande potencial econômico. Em regiões da Colômbia onde ocorre produção de uchuva, é necessário considerar e desenvolver o processamento alternativo do fruto, já que as perdas na colheita e pós-colheita da uchuva no pais, pode chegar a até $45 \%$ da produção total. Neste trabalho foi produzida, polpa, geleia e néctar de uchuva ecotipo Colômbia com frutos ralhados e macios, do municípios Ventaquemada e Cienaga no departamento de Boyacá. Os resultados deste trabalho têm aplicações para as regiões produtoras de uchuva na Colômbia que sofrem perda por rachadura e amolecimento do fruto. Três formulações de cada produto foram realizados fazendo variar o teor de polpa do produto final, foi realizada caracterização físico-química, microbiológica e sensorial. Finalmente, foi calculado o custo de cada produto mais aceitável para o painel. Os resultados mostraram que ao nível microbiológico e físico-químico, os produtos cumprem as normas. A nível sensorial, a polpa mais aceita foi a pasteurizado com açúcar, o néctar mais aceito foi a formulação com $20 \%$ de polpa e geleia mais aceita foi a formulação com polpa de $50 \%$. O custo para processar $1 \mathrm{~kg}$ de frutas foi de 3,11USD para polpa, 3,36USD para néctar e 3,66USD para geleia.

Palavras-chave: uchuva, ralhado, subprodutos, inocuidade.

\section{Introducción}

Las exportaciones colombianas de frutas exóticas, entre ellas la uchuva, datan de hace pocos años, no más de dieciseis, cuando por iniciativa privada, la GTZ (Corporación Técnica Alemana) y Proexport Colombia iniciaron en forma aventurada un sondeo del mercado alemán con el fin de evaluar las posibilidades de una gama, por demás amplia, de frutas exóticas colombianas.

Las exportaciones de uchuva representan el segundo renglón más importante de exportación de frutas colombianas después del banano. Colombia es el principal productor de esta fruta le siguen Sudáfrica, Zimbabwe, Kenya, Ecuador, Perú y Bolivia (Mazorra, 2006). En el 2012 se alcanzó una producción de 6800 toneladas de uchuva por un valor de 26 millones de dólares. La uchuva es una de las frutas exóticas colombianas de mayor éxito en el mercado internacional, principalmente en países perteneciente a la Unión Europea.

Los principales departamentos productores de uchuva ecotipo Colombia son: Boyacá, Cundinamarca, Antioquia y Valle del Cauca, con un crecimiento anual de hectáreas sembradas promedio 
de $40 \%$ entre los años de 1998 a 2012 y un crecimiento de producción en toneladas de $37 \%$ en el mismo periodo de tiempo ( $\mathrm{CCl}, 2013)$.

De la uchuva se comercializa el fruto con capacho, formado por cinco sépalos, que lo protege contra insectos, pájaros, patógenos y condiciones climáticas extremas, o el fruto únicamente, que es una baya carnosa en forma de globo, con un diámetro que oscila entre 1,25 y $2,5 \mathrm{~cm}$ y un peso entre 4 y $10 \mathrm{~g}$. En Colombia esta planta crece entre 1500 y 3000 m.s.n.m. (Fischer, 2005).

Los problemas característicos que causan pérdidas en cosecha y poscosecha del cultivo de uchuva son los siguientes: rajaduras, hongos en el cáliz, hongos en el fruto, ablandamiento, pudrición y cambios sensoriales (Angulo, 2005). Dado que debe obtenerse un producto inocuo, es necesario garantizar que la materia prima que ingresa al procesamiento esté libre de problemas físicos, químicos y microbiológicos; por consiguiente se deben procesar frutos libres de pudriciones y hongos. Lo anterior indica que conviene procesar frutos blandos y rajados (Brito, 2002).

En Colombia, entre el 10 y $15 \%$ de la producción total de uchuva adolece de rajado de fruto (CIAA, 2003), lo cual ocasiona reducción del ingreso para el productor. Las empresas exportadoras descartan cerca del $20 \%$ de fruta por esta causa, pero en épocas de precipitación puede alcanzar hasta el 45\% (Fischer, Herrera \& Almanza, 2011). El mercado exige fruta sana, especialmente para consumo en fresco y exportación. Los frutos rajados son rechazados y castigados en precio, debido a que el rajado promueve la aparición de hongos y bacterias durante el almacenamiento y transporte, acortando la vida útil de la fruta (Castro, Rodriguez, \& Vargas, 2008).

El rajado o agrietamiento del fruto es un término generalmente aplicado a ciertos desordenes fisiológicos, que se expresan como una fractura de la cutícula o epidermis. Esta fractura puede ser visible y se extiende dentro de la pulpa del fruto. El factor causante del rajado más reportado por diversos autores es el suministro de agua a la planta, el cual puede ocasionar fluctuaciones en el potencial hídrico del fruto (Kamimura, Yoshikawa \& Ito,1972; Peet \& Willits, 1995), así como la interacción de microelementos como Boro, Calcio y Cobre en la nutrición de la planta (Camacho, 2005). La uchuva tiene cierta predisposición al rajado por ser una baya jugosa con una epidermis muy delgada.

Actualmente se están desarrollando técnicas de procesamiento de uchuva que faciliten derivados estables y de agradables características sensoriales. Estos derivados emplean la uchuva como materia prima, que sin estar alterada, es apta para su empleo en la elaboración de productos como pulpas, néctares, mermeladas, deshidratados, salsas, compotas, helados, conservas y otros (Camacho, 2005). Sin embargo, no se cuenta con estudios donde se aproveche el fruto rajado y blando para su procesamiento.

\section{Materiales y métodos}

El trabajo de investigación se llevó a cabo en las instalaciones del laboratorio de poscosecha de la Unidad de Investigación y Extensión Agroindustrial de la Universidad Pedagógica y Tecnológica de Colombia, sede Duitama.

\section{Materia prima}

Se utilizó uchuva rajada y blanda, ecotipo Colombia, en un estado de maduración número 6 según la carta de color (Norma Técnica Colombiana 4580), proveniente de ASOPROCIEN (Asociación de Productores de uchuva del municipio de Ciénega) y de PROCOAVEN (Productores de uchuva del municipio de Ventaquemada) en el departamento de Boyacá- Colombia. Fueron cosechadas e inmediatamente transportadas al laboratorio en contenedores con hielo seco y barrera a la luz y el calor. 


\section{Obtención de subproductos de uchuva}

Para la preparación de la pulpa, la fruta se lavó y desinfectó, luego se realizó el escaldado con agua para seguir con el despulpado. Se elaboró pulpa cruda (sin tratamiento térmico y sin azúcar), pulpa natural pasteurizada (tratamiento térmico a 72으 por 5 minutos y sin azúcar) y pulpa pasteurizada con azúcar. Finalmente, se empacaron y se almacenaron en condiciones de refrigeración $\left(2^{\circ} \mathrm{C}\right)$ para la caracterización fisicoquímica, microbiológica y sensorial (Ramírez, 2006).

En la obtención de néctar, se utilizó pulpa cruda previamente elaborada y fue sometida a un proceso de filtrado y clarificación con la adición de gelatina sin sabor al $1 \% \mathrm{p} / \mathrm{p}$ con el fin de remover compuestos fenólicos responsables de la astringencia. Luego, se elaboraron tres formulaciones diferentes de néctar, variando la participación de pulpa en el néctar así: $20 \%$, 30\% y 40\%. Se mezclaron previamente ingredientes en polvo: azúcar, espesante (CMC) y ácido cítrico; seguido por la incorporación de ingredientes líquidos: agua y pulpa clarificada. Una vez homogeneizada la mezcla, las formulaciones se sometieron a tratamiento térmico de $72^{\circ} \mathrm{C}$ durante 5 minutos y fueron envasadas y almacenadas a 4ㄷ (Rodríguez, Pulido \& Alba, 2010).

Para la elaboración de mermelada, se utilizó pulpa cruda previamente elaborada, se sometió a cocción adicionándole gelificante (pectina rápida), azúcar y ácido cítrico según los ingredientes de cada formulación variando el contenido de pulpa así: $40 \%$, $50 \%$ y $60 \%$. Luego se dejó concentrar el azúcar hasta alcanzar 68Bix, se envasó en empaques de vidrio y se almacenaron a condiciones ambientales $\left(18^{\circ} \mathrm{C}\right)$ (Colquichagua \& Ortega, 2005).

\section{Caracterización fisicoquímica}

El pH se determinó usando potenciómetro digital marca TESTO 230 con una precisión de 0,11, por inmersión del electrodo en la muestra, previa calibración con soluciones tampón de $\mathrm{pH}$ 2, 4, 7 y 10 a 25드 (AOAC, 1984). Los sólidos solubles se determinaron midiendo el índice de refracción de las muestras de frutas en un refractómetro digital, a 20C (AOAC, 1990). La acidez titulable se determinó según el método oficial 942.15 (AOAC, 1990). La humedad se determinó con la balanza de humedad marca Cittizon. MB 200.

\section{Caracterización microbiológica}

El análisis microbiológico se realizó según los valores de referencia consignados en la resolución 15789/84 y 7992/91 del Ministerio de Salud de Colombia. Se realizó de acuerdo a la metodología oficial de la AOAC (1984) recuento de microorganismos mesofílicos con el método de recuento en placa: AOAC 966.23.C, coliformes fecales y coliformes totales con el método de tubos múltiples fermentativos: AOAC 966.24 y recuento de hongos y levaduras con el método de recuento en placa: AOAC 997.02.

\section{Panel Sensorial}

El panel se realizó mediante pruebas de aceptabilidad con 16 panelistas no entrenados. Se utilizó una prueba de ordenamiento de rangos para obtener una indicación de la muestra de pulpa, néctar y mermelada con la textura, sabor y color más aceptable (Watts, Yllimaki, Jeffery \& Elias, 1992). A los panelistas se les pidió ordenar las muestras de acuerdo a su aceptabilidad, y se les mencionó evitar clasificar dos muestras en la misma posición, debiendo dar un valor diferente a cada muestra, incluso si les parecía similar. Se asignó un valor de 1 a la muestra más aceptable, un valor de 2 a la muestra que le sigue en grado de aceptabilidad y un valor de 3 a la que tenía la textura, sabor y color menos aceptable.

Para el análisis de datos, se sumaron el total de los valores de posición asignados a cada muestra; a continuación, se determinaron las diferencias significativas con un nivel de significancia del 95\% entre muestras, comparando los totales de los valores de posición de todos los posibles pares de muestras utilizando la prueba de Friedman (Newell \& MacFarlane, 1987). 
Una vez determinadas las formulaciones más aceptadas de cada subproducto, se realizó el cálculo de costo total de producción, teniendo como referencia los costos de empresas del departamento de Boyacá, procesadoras de pulpas, néctares y mermeladas con capacidad similar a la propuesta en esta investigación. La base de cálculo fue 360 $\mathrm{kg}$ de fruta, este valor corresponde al promedio de uchuva que se pierde, debido al rajado y ablandamiento, por cada tonelada cultivada en Colombia (R. Fonseca, entrevista personal, 2013) y que se procesa mensualmente por una asociación de productores de uchuva.

\section{Resultados y discusión}

En la Tabla 1 se presentan las características fisicoquímicas de uchuva rajada y blanda ecotipo Colombia obtenidas en la presente investigación, en ésta se puede observar que la fruta utilizada como materia prima presenta características adecuadas para su transformación. Entre las más importantes está su elevado rendimiento en pulpa (70\%) cercano al de guayaba (74\%), mora (78\%) y superior a guanábana $(60 \%)$, mango $(50 \%)$ y piña (50\%) (Camacho, 2005). Estos valores dependen de las variedades y ecotipos. Sin embargo, para uchuva ecotipo Colombia, cultivada en diferentes regiones del país, representan características apreciables en el mercado internacional. Las diferencias entre resultados y valores de referencia radican en que la fruta rajada y blanda ha tenido un proceso de deshidratación debido a grietas en la epidermis que dejan expuesta la pulpa al ambiente y a una consecuente pérdida de agua.

En promedio, la fruta posee $13^{\circ}$ Brix, correspondientes a los sólidos solubles de la uchuva. Según Torres, Cooman \& Fischer, 2004. el contenido de sólidos solubles totales diluidos en el jugo del fruto están constituidos de un $80 \%$ a $95 \%$ por azúcares. Los ${ }^{\circ}$ Brix presentados en uchuva rajada son adecuados a su estado de madurez y apropiados para los balances de masa, dado que a menos Brix, mayor cantidad de azúcar se debe emplear en la elaboración de mermeladas, néctares, salsas, refrescos y otros (Almanza \& Espinosa, 1995).

El pH fue 3.7, demostrando que la uchuva es una fruta ácida, impidiendo el fácil crecimiento de microorganismos patógenos (Vaclavik, 2002). Además en la elaboración de mermeladas y bocadillos este valor favorece la natural gelificación. La acidez titulable de uchuvas, de buena calidad, tiene porcentajes entre 1,3\% y 1,6\% (Flórez, Fischer \& Sora, 2000), es un valor atractivo para los procesadores lográndola emplear en la preparación de néctares, obteniendo un equilibrio de sabores dulce-ácido adicionando agua y azúcar (Marín, 2009).

Tabla 1. Resultados de la caracterización fisicoquímica de la uchuva utilizada como materia prima

\begin{tabular}{lcc}
\hline \multicolumn{1}{c}{ Parámetros } & Resultado & Valor de referencia \\
\hline Pulpa g/100 g fruta & $65 \pm 0,2$ & 70 \\
\hline Cáscara g/100g de fruta & $3,2 \pm 0,1$ & 3,5 \\
\hline Semillas/100 g de fruta & $24 \pm 0,15$ & 26,5 \\
\hline $\begin{array}{l}\text { oBrix } \\
\text { (Estado de madurez 6. NTC 4580) }\end{array}$ & $13 \pm 0,2$ & 13 \\
pH & $3,7 \pm 0,3$ & $3,5-3,7$ \\
Acidez Titulable (\% ácido cítrico) & $1,4 \pm 0,1$ & $1,3-1,6$ \\
\hline Humedad (\%bh) & $87 \pm 0,08$ & $86-88$ \\
\hline
\end{tabular}

Nota: valor de referencia de la resolución 15789/84 y 7992/91 del Ministerio de Salud de Colombia 
La Tabla 2 presenta la caracterización fisicoquímica de subproductos de uchuva rajada y blanda, en ésta se puede observar que las muestras están entre los parámetros de referencia estipulados por la normatividad legal vigente de Colombia. Se pueden obtener varios tipos de pulpa de uchuva rajada. El más común es pulpa cruda congelada, la cual ha sido simplemente congelada después de su obtención (Camacho, 2002). Ésta tiene varias ventajas: conserva sus características nutricionales y sensoriales intactas, siempre y cuando no sea sometida a demasiada aireación durante el despulpado.
Adicionalmente se puede elabora pulpa natural pasterizada y edulcorada pasterizada.

Las tres formulaciones de néctares presentaron algunas variaciones en ${ }^{\circ}$ Brix y acidez, lo cual otorga diferentes niveles de sabor dulce y ácido, pero con características de color y consistencia muy similares. En la elaboración de mermelada, se observó la formación característica del gel por la presencia del ácido natural de la fruta. Adicionalmente, el color naranja de la fruta se mantuvo estable en el tratamiento térmico.

Tabla 2. Resultados de la caracterización fisicoquímica de subproductos de uchuva

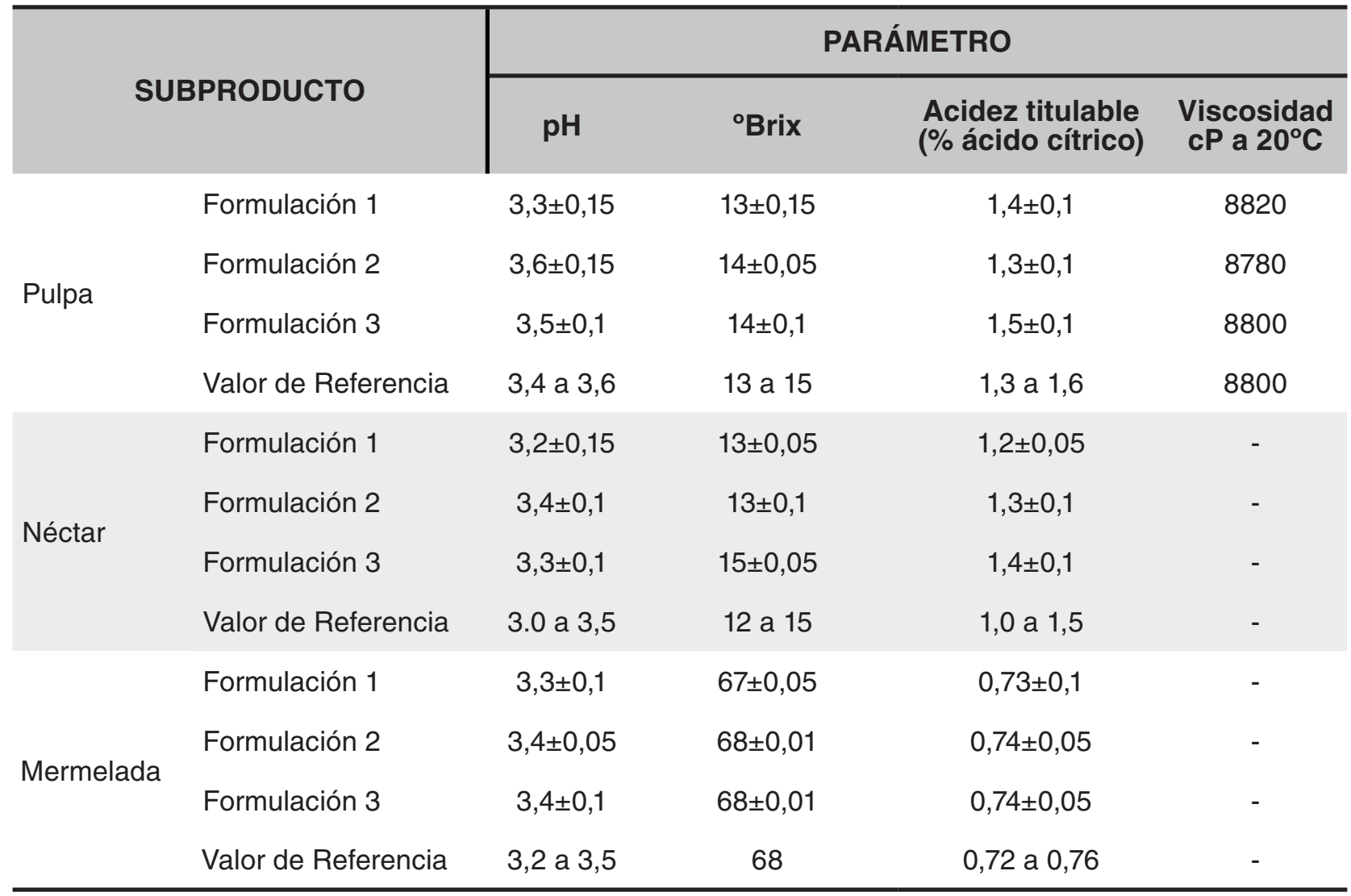

Nota: valor de referencia de la resolución 15789/84 y 7992/91 del Ministerio de Salud de Colombia

Respecto a la caracterización microbiológica de subproductos, se observa en la Tabla 3 que los valores de las muestras analizadas cumplen con los requisitos microbiológicos, según lo recomendado como valor de referencia por la normatividad legal vigente para pulpas, néctares y mermeladas. Para obtener productos inocuos con baja carga bacteriana, es necesario mantener condiciones de higiene y saneamiento durante el proceso, equipos, utensilios y personal (Jay, 2002). Debido al uso de uchuva rajada expuesta al ambiente, se deben evitar periodos de tiempo largos entre cosecha, poscosecha y transformación para evitar contaminación de la fruta (Leterme, Buldgen, Estrada \& Londoño, 2006). 
Todas las formulaciones de subproductos, excepto la pulpa cruda congelada, se sometieron a tratamientos térmicos garantizando la reducción de microrganismos a un nivel seguro. La pulpa cruda congelada, aunque no fue sometida a tratamiento térmico, resultó con valores de microorganismos aceptables para su consumo. Se debe resaltar el hecho de que todos los subproductos elaborados fueron preparados $100 \%$ naturales, sin adición de conservantes y/o colorantes.

Tabla 3. Resultados de la caracterización microbiológica de subproductos de uchuva

\begin{tabular}{|c|c|c|c|c|c|c|}
\hline \multirow{2}{*}{ 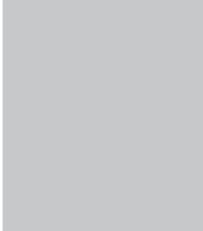 } & \multirow{2}{*}{\multicolumn{2}{|c|}{ SUBPRODUCTO }} & \multicolumn{4}{|c|}{ PARÁMETRO } \\
\hline & & & $\begin{array}{l}\text { Aerobios } \\
\text { mesófilos } \\
\text { (UFC/g) }\end{array}$ & $\begin{array}{l}\text { Coliformes } \\
\text { Totales } \\
\text { (NMP/g }\end{array}$ & $\begin{array}{c}\text { Coliformes } \\
\text { fecales } \\
\text { (NMP/g) }\end{array}$ & $\begin{array}{l}\text { Mohos y } \\
\text { levaduras } \\
\text { (UFC/g) }\end{array}$ \\
\hline \multirow{5}{*}{ Pulpa } & \multicolumn{2}{|c|}{ Formulación 1} & 5000 & $<9$ & $<3$ & $<100$ \\
\hline & \multicolumn{2}{|c|}{ Formulación 2} & $<20$ & $<3$ & $<3$ & $<100$ \\
\hline & \multicolumn{2}{|c|}{ Formulación 3} & $<20$ & $<3$ & $<3$ & $<100$ \\
\hline & \multirow{2}{*}{$\begin{array}{l}\text { Valor de } \\
\text { Referencia }\end{array}$} & $\begin{array}{l}\text { Pulpa cruda } \\
\text { congelada }\end{array}$ & $\begin{array}{c}20.000- \\
50.000\end{array}$ & $\leq 9$ & $<3$ & $\begin{array}{l}1.000- \\
3000\end{array}$ \\
\hline & & $\begin{array}{l}\text { Pulpa } \\
\text { pasterizada }\end{array}$ & $1.000-3.000$ & $<3$ & $<3$ & $100-200$ \\
\hline \multirow{4}{*}{ Néctar } & \multicolumn{2}{|c|}{ Formulación 1} & 50 & $<3$ & $<3$ & $<100$ \\
\hline & \multicolumn{2}{|c|}{ Formulación 2} & 50 & $<3$ & $<3$ & $<100$ \\
\hline & \multicolumn{2}{|c|}{ Formulación 3} & 50 & $<3$ & $<3$ & $<100$ \\
\hline & \multicolumn{2}{|c|}{ Valor de Referencia } & $1.000-3.000$ & $9-29$ & 3 & $100-200$ \\
\hline \multirow{4}{*}{ Mermelada } & \multicolumn{2}{|c|}{ Formulación 1} & $<10$ & $<3$ & $<3$ & $<10$ \\
\hline & \multicolumn{2}{|c|}{ Formulación 2} & $<10$ & $<3$ & $<3$ & $<10$ \\
\hline & \multicolumn{2}{|c|}{ Formulación 3} & $<10$ & $<3$ & $<3$ & $<10$ \\
\hline & \multicolumn{2}{|c|}{ Valor de Referencia } & $100-300$ & $<3$ & $<3$ & $20-50$ \\
\hline
\end{tabular}

Nota: valor de referencia de la resolución 15789/84 y 7992/91 del Ministerio de Salud de Colombia

En cuanto al análisis sensorial, los resultados del panel indican que la pulpa más aceptada sensorialmente fue la formulación 3: pulpa pasterizada con azúcar. Estadísticamente, la prueba de Friedman indicó que los panelistas no encontraron diferencias sensoriales entre la formulación 1 y 2, es decir la pulpa cruda y la pulpa pasterizada. El néctar más aceptado fue la formulación 1: néctar con $20 \%$ de pulpa, estadísticamente, los panelistas encontraron diferencias sensoriales entre las tres formulaciones, es decir aunque el néctar más aceptado fue la formulación 1, las otras dos formulaciones poseían características sensoriales diferentes. La mermelada más aceptada fue la formulación 2: mermelada con $50 \%$ de pulpa y las formulaciones con diferencias significativas fueron la $1(40 \%$ pulpa) y 3 (60\% pulpa), por lo tanto, al no detectar diferencias significativas entre la formulación 1 y 2, debería optarse por la elaboración de mermelada con menor participación de pulpa, disminuyendo así los costos de materias primas. 
En la Tabla 4 se presenta el costo total de producción para el procesamiento de $360 \mathrm{~kg}$ de uchuva rajada y blanda, correspondiendo al valor promedio de uchuva que se pierde debido al rajado y ablandamiento por cada tonelada cultivada en Colombia. Según las formulaciones de subproductos más preferidas por el panel, se han incluido equipos y otros elementos para realizar las operaciones básicas para el procesamiento de subproductos de uchuva bajo un esquema de procesamiento por baches (Henley \& Roson, 2002).

Tabla 4. Costo total de producción para subproductos de uchuva

\begin{tabular}{l|ccc}
\hline \multirow{2}{*}{ ELEMENTO } & PULPA & NÉCTAR & MERMELADA \\
\cline { 2 - 4 } & COSTO (USD) & COSTO (USD) & COSTO (USD) \\
\hline Insumos & 210 & 306 & 339 \\
Mano de obra & 120 & 120 & 120 \\
\hline Equipos & 570 & 612 & 641 \\
Otros elementos & 223 & 173 & 219 \\
de trabajo & 1123 & 1211 & 1319 \\
Total & 3,11 & 3,36 & 3,66 \\
\hline Total/ kg fruta & & & \\
\hline
\end{tabular}

Fuente: información de empresas de Boyacá, procesadoras de pulpas, néctares y mermeladas

\section{Conclusiones}

Los resultados tienen aplicaciones para las regiones de Colombia donde se produzca uchuva con problemas de pérdidas por rajado y ablandamiento del fruto en las etapas de cosecha y poscosecha.

La uchuva rajada resultó ser apta para el procesamiento de mermelada dado que cumplió las características fisicoquímicas, sensoriales y de calidad recomendadas para el consumo humano.

La pulpa, néctar y mermelada son opciones para el aprovechamiento de la fruta rajada y blanda, agregando valor a frutos de uchuva que se comercializan generalmente en fresco.
El panel sensorial es una herramienta indispensable en el desarrollo de productos procesados, puesto que indica la aceptación que tendrá el alimento en el mercado objetivo para lo cual se debe tener cuidado en el diseño de las pruebas, realizar el análisis de datos y escoger los panelistas de tal manera que se ajuste a las necesidades del estudio y que sea una muestra representativa del mercado objetivo.

El costo total para procesar $360 \mathrm{~kg}$ de uchuva rajada y blanda en la obtención de pulpa es de 1123 USD, 1211 USD en la elaboración de néctar y 1319 USD en el procesamiento de mermelada. 


\section{Agradecimientos}

Los autores expresan su agradecimiento al Departamento de Investigaciones UPTC, Grupo CERES. Proyecto de Capital Semilla número 890.

\section{Literatura citada}

1. Almanza, P. \& Espinosa, C. (1995). Desarrollo morfológico y análisis físico químico de frutos de uchuva Physalis peruviana L. para identificar el momento óptimo de cosecha. Facultad de Ciencias Agropecuarias, vol. Especialista en frutales en clima frío (pp. 83). Tunja: Universidad Pedagógica y Tecnológica de Colombia.

2. Angulo, R. (2005). Uchuva - El cultivo. Centro de Investigaciones y Asesorías Agroindustriales (CIAA). Universidad de Bogotá Jorge Tadeo Lozano. Bogotá. 78 p.

3. AOAC. Association of Official Analytical Chemists. (1984). Official Methods of Analysis. 13th ed. Ed. S. Williams. Washington D.C. 527 p.

4. AOAC. Association of Official Analytical Chemists. (1990). Official Methods of Analysis. 15th ed. Ed. S. Williams. Washington D.C. 535 p.

5. Brito, D. (2002). Producción de uvilla para exportación. Agroexportación de productos no tradicionales (p.10). Quito, Ecuador: Fundación Aliñambi.

6. Camacho, G. (2002). Transformación y Conservación de Frutas. Instituto de Ciencia y tecnología de Alimentos. Universidad Nacional de Colombia. Curso virtual recuperado de: http://www.virtual.unal.edu.co/cursos/ agronomia/2006228/index.html

7. Camacho, G. (2005). Procesamiento de uchuva. pp. 129-145. En: Flórez, V.J., G. Fischer y A.D. Sora. 2000. Producción, poscosecha y exportación de la uchuva (Physalis peruviana L.). Editorial: Unibiblos, Universidad Nacional de Colombia, Bogotá. 175 p.

8. Castro, A., Rodriguez, L., \& Vargas, E. (2008). Dry gooseberry (Physalis peruviana $L$ ) with a pretreatment of osmotic dehydration. Vitae - Revista de la Facultad de Química Farmacéutica, 15(2), 226-231.

9. CIAA Centro de Investigaciones y Asesorías Agroindustriales. (2003). Evaluación y desarrollo de un modelo de producción de lulo y uchuva bajo condiciones de invernadero. 40 Informe Técnico a Colciencias. Universidad de Bogotá Jorge Tadeo Lozano. 65 p.

10. Colquichagua, D. \& Ortega, E. (2005). Procesamiento de mermeladas de frutas nativas. Editorial punto impreso. Lima. 27p.

11. CCI Corporación Colombia Internacional. (2013). Inteligencia de mercados. Recuperado de: http://www.agronet.gov.co/www/docs_agronet/2006327162612_uchuva_CCl_actualizaci\%C3\%B3n.pdf
12. Fischer, G. (2000). Crecimiento y desarrollo. pp. 9-26. En: Flórez, V.J., G. Fischer y A.D. Sora (eds.). 2000. Producción, poscosecha y exportación de la uchuva (Physalis peruviana L.). Editorial: Unibiblos, Universidad Nacional de Colombia, Bogotá. 175 p.

13. Fischer, G. (2005). El problema del rajado del fruto de uchuva y su posible control. pp. 55-82. En: Fischer, G., D. Miranda., Piedrahita, W \& Romero, J. Avances en cultivo, poscosecha y exportación de la uchuva (Physalis peruviana L.) en Colombia. Editorial: Unibiblos. Universidad Nacional de Colombia, Bogotá. 221p.

14. Fischer, G., Herrera, A. \& Almanza, P. (2011). Cape gooseberry (Physalis peruviana L.). pp. 374-396. En: Yahia, E.M. Postharvest biology and technology of tropical and subtropical fruits. Vol. 2. Acai to citrus. Woodhead Publishing, Oxford, U.K. 532 p.

15. Flórez, V.J., G. Fischer \& A.D. Sora. (2000). Producción, poscosecha y exportación de la uchuva (Physalis peruviana L.). Editorial: Unibiblos, Universidad Nacional de Colombia, Bogotá. 175 p.

16. Henley, E \& Roson, M. (2002). Cálculo de Balances de Materia y Energía. 1a edición. Editorial Reverté S.A. México.

17. Jay, M. (2002). Microbiología Moderna de los Alimentos. 4⿳a edición. Editorial Acribia. Zaragoza. España Pg. 342- 345. $475 \mathrm{p}$.

18. Kamimura, S., H. Yoshikawa \& K. Ito. (1972). Studies on fruit cracking in tomatoes. Horticultural Research. Station of the Ministry of Agriculture and Forestry, Morioka. Series C. No. 7.

19. Leterme, P., Buldgen, A., Estrada, F., \& Londoño, A. M. (2006). Mineral content of tropical fruits and unconventional foods of the Andes and the rain forest of Colombia. Food Chemistry, 95(4), 644-652.

20. Marín, Z. (2009). Viabilidad de desarrollo de uchuva (Physalis peruviana L.) mínimamente procesada enriquecida con microorganismos probióticos a partir de la Ingeniería de Matrices. Facultad de Ciencias Agropecuarias. Maestría en Ciencia y Tecnología de Alimentos (pp. 152). Medellín: Universidad Nacional de Colombia.

21. Mazorra, M. (2006). Aspectos anatómicos de la formación y crecimiento del fruto de uchuva Physalis peruviana (Solanaceae). Acta Biológica Colombiana, 11(1), 69-81.

22. Newell, G.J. \& MacFarlane, J.D. (1987). Expanded Tables for Multiple Comparison Procedures in the Analysis of Ranked Data. In Journal of Food Science, 52, 1721-1725.

23. Peet, M \& D. Willits. (1995). Role of excess water in tomato fruit cracking. Hort Science 30(1), 65-68.

24. Ramírez, R. (2006). Tecnología de frutas y hortalizas. Universidad Nacional Abierta y a Distancia (UNAD). Bogotá. 312p. 
25. Rodríguez, L., Pulido, N \& Alba, J. (2010). Formulación de Néctar de Marañón (Anacardium Occidentale L) usando la metodología de superficie de respuesta para optimizar la aceptación sensorial y la actividad antioxidante. Revista de la Asociación Colombiana de Ciencia y Tecnología de Alimentos 20(24), 47-52.

26. Torres, C., A. Cooman \& Fisher, G. (2004). Determinación de las causas del rajado del fruto de uchuva (Physalis peruviana L.) bajo cubierta: I. Efecto de la variación en el balance hídrico. Revista Agronomía Colombiana, 22(2), 140-146.
27. Vaclavik, V. (2002). Fundamentos de ciencia de los alimentos. 1aㅡ Edición. Editorial Acribia. Zaragoza. España. 483p.

28. Watts, B., Ylimaki, G., Jeffery, L \& Elìas, L. (1992) Métodos Sensoriales básicos para la evaluación de alimentos. Centro Internacional de Investigaciones para el Desarrollo (CIID). Oficina Regional para América Latina y el Caribe. Uruguay. 170 p. Recuperado de: http://idl-bnc.idrc.ca/dspace/bitstream/10625/12666/1/89276.pdf. 